\title{
Skin Hyperpigmentation, CTCAE
}

National Cancer Institute

\section{Source}

National Cancer Institute. Skin Hyperpigmentation, CT CAE. NCI Thesaurus. Code C143835.

A disorder characterized by darkening of the skin due to excessive melanin deposition. 